\title{
An Evolutionary Computational Approach for the Problem of Unit Commitment and Economic Dispatch in Microgrids under Several Operation Modes
}

\author{
L. Alvarado-Barrios $^{1, *(\mathbb{D})}$, A. Rodríguez del Nozal ${ }^{1}$, A. Tapia ${ }^{1} \mathbb{D}$, J. L. Martínez-Ramos ${ }^{2}{ }^{\mathbb{D}}$ and \\ D. G. Reina ${ }^{3}$ (D)
}

1 Departamento de Ingeniería, Universidad Loyola Andalucía, 41014 Seville, Spain; alvarordn@hotmail.com (A.R.d.N.); atapia@uloyola.es (A.T.)

2 Electrical Engineering Department, University of Seville, 41092 Seville, Spain; jl.martinez.ramos@gmail.com

3 Electronic Engineering Department, University of Seville, 41092 Seville, Spain; dgutierrezreina@us.es

* Correspondence: lalvarado@uloyola.es

Received: 17 April 2019; Accepted: 30 May 2019; Published: 4 June 2019

\begin{abstract}
In the last decades, new types of generation technologies have emerged and have been gradually integrated into the existing power systems, moving their classical architectures to distributed systems. Despite the positive features associated to this paradigm, new problems arise such as coordination and uncertainty. In this framework, microgrids constitute an effective solution to deal with the coordination and operation of these distributed energy resources. This paper proposes a Genetic Algorithm (GA) to address the combined problem of Unit Commitment (UC) and Economic Dispatch (ED). With this end, a model of a microgrid is introduced together with all the control variables and physical constraints. To optimally operate the microgrid, three operation modes are introduced. The first two attend to optimize economical and environmental factors, while the last operation mode considers the errors induced by the uncertainties in the demand forecasting. Therefore, it achieves a robust design that guarantees the power supply for different confidence levels. Finally, the algorithm was applied to an example scenario to illustrate its performance. The achieved simulation results demonstrate the validity of the proposed approach.
\end{abstract}

Keywords: microgrids; Unit Commitment; Economic Dispatch; Genetic Algorithm

\section{Introduction}

The deregulation process in the energy sector has encouraged a transformation of the Electric Power Systems (EPS). EPS are defined as networks of electrical components deployed to supply, transfer, store and use electric power. Nevertheless, in the last decades, EPS are changing their classical scheme, which has been traditionally characterized by a centralized top-down operated architecture, to a distributed model. This new scheme is strongly characterized by an increasing integration of Renewable Energy Sources (RES) [1,2]. In this scenario of transformation, microgrids, which are defined as coordinated aggregations of loads and generation resources in medium and low voltage grids [3], play a fundamental role [4,5]. This is mainly due to their capability of integrating and exploiting RES, together with additional benefits, such as enhancing the entrance of electric vehicles [6]. Microgrids are generally composed by a certain set of loads, conventional and renewable generation units, and storage units, being these last components operated as independent and controllable units [7,8], either connected or disconnected from the grid [9].

The operation of a microgrid is performed by using Energy Management Systems (EMS) [10], which are responsible for commanding the local generation, storage and flexible demand resources. 
EMS dispatches the active energy from RES and control the load consumption in the most optimal way. This management is normally carried out on the basis of an established criterion [11]. In this context, the problem of Unit Commitment (UC) represents an important tool to study the optimal scheduling of generation resources [12], contributing not only to improve the stability and reliability of the microgrid, but also reducing the environmental impact and the costs associated to the operation [13]. The UC consists of finding the most suitable schedule for coordinating the different generation and storage units to achieve a target objective, such as minimizing the cost or maximizing the benefits.

Once the UC is solved, the Economic Dispatch (ED) is responsible for assigning the generation reference to the programmed units, satisfying the power demand together with the physical constraints of the grid [14]. Sometimes, this problem is tackled considering the possible uncertainty in the power generated of the RES or the demand. Thus, this combined problem aims to deal with the variability of both the RES and the demand [1,7].

In general, the uncertainty in the UC problem is represented by the formulation of a set of scenarios, which are made on the basis of the predictions of the demand and the RES generation. In contrast, deterministic approaches are commonly used, where a single scenario is considered $[15,16]$. Given these features, stochastic approaches are crucial to address an optimal management of microgrids [17], which has motivated several studies in the literature [18-20]. These approaches contribute to the robustness of the solution, making them more appropriate to microgrid implementations.

Finally, it is worth pointing out the complexity of the combined UC and ED problem [21]. The schedule of the dispatchable generation units together with the management of the ESS, with the additional consideration of uncertainties in the demand forecasting generates a non-convex mathematical problem, which combines strong non-linearities and mixed integer formulation. Although some authors have proposed to linearize the objective functions, it introduces inaccuracies into the resulting operation cost. In addition, such linearization should be done at each operation point, which can result in a slow iterative procedure. Therefore, such approach can suffer from convergence and scalability problems for high dimensional scenarios. Similar to linear programming based approaches, metaheuristic algorithms such as evolutionary algorithms allow dealing with non-linearities in an effective way since the search engine is based on genetic operations (selection, crossover, and mutation) among potential solutions. Given these characteristics of the combined UC and ED problem, solving it in the most optimal way constitutes itself a challenge. Furthermore, it is important to note that small deviations in the solution might imply noticeable effects in the operational and maintenance costs of the microgrid, also affecting the level of emissions. For these reasons, the use of meta-heuristic approaches is of particular relevance.

The main contributions of this work are summarized:

- A new methodology is introduced to deal with the generation schedule problem of a microgrid where the demand forecasting is affected by uncertainties. This approach ensures the robustness of the algorithm at the expense of affecting the operational and environmental cost of the installation.

- The microgrid model includes several operation modes optimizing different aspects of the microgrid such as power cost, emissions, and robust operation with respect to the demand variability. Combined operation modes, where a combination of objective functions are used, are considered.

- An evolutionary approach for solving the UC and ED problems in microgrids is presented.

The paper is organized as follows. Section 2 presents the related work of current approaches that have addressed EMS in microgrids. Section 3 introduces the stochastic UC and ED problems in a microgrid. In Section 4, the different components of the microgrid are modeled in detail. Section 5 presents the operation modes of the considered microgrid. In Section 6, an evolutionary computational approach is proposed to address the problem. Section 7 contains the evaluation of the proposed approach through extensive simulations. Finally, in Section 8 some conclusions and remarks are drawn together with future work lines. 


\section{Related Work}

An extensive review of the objectives, constraints and algorithms used in EMS for microgrids is addressed in [18], where the authors provided a brief summary of the main strategies used to tackle this problem: linear, non-linear, stochastic, dynamic and non-differential programming. In addition, a classification of the different optimization techniques reported in the literature is presented.

The UC problem is generally classified as Mixed Integer Non-Linear Programming (MINLP) [19,22,23]. An illustrative work is [22], where the authors proposed a profit-based UC problem with non-linear emissions penalty. This penalty has been transformed through a mixed-integer formulation, while the non-linearities of the fuel cost function are addressed by means of a piece-wise linearization. The proposed algorithm is used to solve several architectures, exhibiting good results. A very similar strategy is used in [23], where numerical results confirm the robustness of the approach. The authors of [24] proposed a MILP to determine a fair cost distribution among smart homes with microgrid, obtaining noticeable cost reductions and fair cost distribution among multiple homes under a set of test scenarios.

Despite of the high efficiency of MILP approaches, it is worth pointing out that the computational cost of these approaches increases drastically with the number of integer variables. To address this drawback, several proposals are discussed. In [25], a multi-step method is used to consider the combinatorial calculations of unit outage events. This method noticeably improves the calculations of the spinning reserve, while reducing the computational burden. The authors of [26] proposed the resolution of different scales separately, by means of a bottom-up approach, keeping track of the error. This strategy strongly reduces the computational time required to find an optimal solution without sacrificing the quality of the results. Therefore, it improves the capability of MILP approaches to address large-scale modelling of urban energy systems. In addition, in [27], the authors proposed an improved MILP to increase the efficiency of the approach for its application to big scale grids, where the results in different scales show a dramatic improvement in comparison with other MILP proposed in the literature. It is also relevant to highlight the capability of these approaches to address the energy optimization in city environments [28]. A relevant work is found in [29], where the scheduling problem of building energy supply is addressed to minimize the overall cost of the energy consumption. Mixed-Integer Quadratic Problem (MIQP) is also used to address the UC problem. An illustrative work can be found in [30], where the authors proposed an improved MIQP, which improves its efficiency by means of relaxation and decoupling techniques.

In addition to both MILP and MIQP approaches, a wide variety of optimization algorithms are proposed in the literature [31,32] to address this problem (see Table 1, where the most relevant works are summarized). The studied works present a wide variety of architectures, generally including controllable RES and operating either isolated or connected to the grid.

An illustrative example can be found in [13], where two optimization algorithms are developed, based on a Genetic Algorithm (GA) and MILP, respectively. Both algorithms are used in a radial low-voltage European microgrid for which the authors formulated a set of objective functions and constraints, attending to the different modes of operation of the microgrid, such as cost-efficiency, grid supporting, reliable isolated operation, eco-friendliness and multi-functional operations. The results demonstrate a better performance of the GA in comparison to MILP. Similarly, in [31], a more complex problem is formulated by considering start-up and shut-down costs associated to the distributed generators (DGs). This approach is used in a microgrid, being the problem addressed by means of a hybrid optimal algorithm, which consists in decomposing the problem into Integer Programming (IP) to solve the UC problem and Non-Linear Programming (NLP) to solve the ED problem. After that, both GA and interior-point algorithms are used to solve the IP and NLP problems, respectively. An Enhanced Genetic Algorithm (EGA) is proposed in [33] to address both the UC and ED problems simultaneously. The performance and effectiveness of the developed GA is verified in a set of case-study optimization scenarios, which are applied to a typical test microgrid. The simulation results demonstrate a good performance (in terms of computational time and robustness) of the optimizer 
under grid-connected operation mode, in contrast with the isolated mode, where the computational resources required increase.

In [34], the minimization of the energy cost is assumed as the objective function for the optimization problem. The problem is addressed by applying an evolutionary approach based on Memory-Based Genetic Algorithm (MGA). The results are compared with two variants of Particle Swarm Optimization (PSO) algorithms, exhibiting a noticeably superior behavior. Similarly, the authors of [35] addressed the same problem by improving a GA with the integration of a Simulated Annealing (SA) algorithm to accelerate the convergence, demonstrating a clear advantage in convergence time.

In [36], the optimal size of the storage systems and the RE micro-sources is studied by means of a fuzzy multi-objective optimization model. The approach is based on a chaos optimization algorithm, which is introduced into a Binary particles Swarm Optimization (BPSO) algorithm, to propose a Chaotic BPSO (CBPSO). Furthermore, some authors proposed the use of Monte Carlo simulations as a tool to generate scenarios. In [37], the non-parametric neural network based prediction intervals (PIs) are implemented for forecast uncertainty quantification of wind power. A GA is then used to solve the Stochastic Unit Commitment (SUC) problem, being the Monte Carlo simulations used to create the scenarios. The simulation results demonstrate a better performance of the CBPSO in comparison with the original BPSO. Similarly, in [32], the uncertainty of demand and wind sources is considered, being modeled by means of absolute percentage error. Demand and wind power scenarios are generated using Monte Carlo simulations. To optimize the UC problem, the priority list method (PL algorithm) [38] is applied.

Table 1. Relevant literature in optimization of microgrids. Objective Function: Operation Cost (CO), Emission Cost (EC), Compensation Cost of Load Shedding (CCLS), network loss (NL), Economical Revenue (ER). Type: Single Objective. Microgrid: Standalone (SA), Grid Connected (GC). Approach: Deterministic Unit Commitment (DUC), Stochastic Unit Commitment (SUC). Strategy: Genetic Algorithm (GA), Enhanced Genetic Algorithm (EGA), Integer Programming (IP), Non-Linear Programming (NLP), Simulated Annealing (SA), Mixed Integer Linear Programming (MILP), Binary Particle Swarm Optimization (BPSO), Chaotic Binary Particle Swarm Optimization (CBPSO).

\begin{tabular}{cccccc}
\hline Ref. & Objective Function & Type & Microgrid & Approach & Strategy \\
\hline$[13]$ & $\min$ OC & SO & SA, GC & DUC & GA, MILP \\
{$[20]$} & $\min \mathrm{f}(\mathrm{OC}, \mathrm{EC}, \mathrm{CCL}, \mathrm{NL})$ & SO & SA & DUC & BPSO \\
{$[31]$} & min OC & SO & GC & DUC & GA + IP (UC), NLP(ED) \\
{$[33]$} & min OC & SO & SA & DUC & EGA \\
{$[34]$} & min OC & SO & SA & DUC & MGA \\
{$[35]$} & $\min$ OC, max ER & SO & SA and GC & DUC & GA + SA \\
{$[36]$} & min EC, min NL & SO & SA & SUC & BPSO, CBPSO \\
{$[37]$} & min GC & SO & GC & DUC, SUC & GA \\
{$[32]$} & $\min$ GC, min SUC + SDC & SO & GC & SUC & PL \\
\hline
\end{tabular}

As steps forward with respect to the previous works, this paper proposes:

- Two main modes of operation are considered: cost effective operation mode, in which the main aim is to minimize the operation and maintenance cost of the overall system; and eco-mode operation mode, in which the objective is to reduce the polluted emissions of the dispatchable units.

- The general model improves the scheduling problem of microgrids by considering different levels of reliability according to the uncertainty of the forecast demand. Thus, the spinning reserve of dispatchable generation units guarantees that the variability of the demand is covered. 


\section{Problem Description}

New types of generation technologies have emerged and have been gradually integrated into the existing power systems in the last decades [39], moving their classical architecture to distributed schemes. These technologies have associated lower emissions and lower operation costs than conventional generators. Examples of this type of technologies are micro-turbines, photo-voltaics and fuel cells, among others. Despite the positive features aforementioned, the application of these distributed architectures have many associated problems that must be deeply studied [40]. This challenge can be partially addressed by microgrids, which are entities that coordinate the Distributed Energy Resources (DER) in a consistently more decentralized way, thereby reducing the control burden on the grid and permitting them to provide their full benefits [41].

Under this new paradigm, a microgrid contains multiple generators that have a wide range of operation modes. Thus, several strategies can be applied to meet the required power demand, which varies from hour to hour over the day [12]. This problem is formally defined as the Unit Commitment (UC) and Economic Dispatch (ED) problems. The general objective of both is to minimize the total operating cost, while satisfying all of the constraints so that a given security level can be met $[14,42,43]$. The UC problem studies which generators must be in operation at certain time instant, while the ED studies, once the generators connected to the grid are known, the power that must be supplied by each of them.

These approaches attend to cover not only to economical factors, but it also considers environmental and technical limitations.

In particular in this paper, we consider the study of the microgrid depicted in Figure 1. This grid contains four generators, being two of them dispatchable generating units. Recall that a dispatchable generating unit refers to sources of electricity that can be used on demand at the request of operators, that is, can be turned on and off, or can adjust their power output according to the needs. Thus, the microgrid under study is composed by a diesel engine (DE) and a microturbine (MT) as dispatchable units, and a wind turbine (WT) and a photo-voltaic plant (PV) as non-controllable RES. Finally, an Energy Storage System (ESS) is also considered. The model of these components, together with the customer demands, is studied in the subsequent sections. Thus, the problem studied is to determine the power supplied by the different generators as well as the power supplied or demanded by the ESS at every time instant, minimizing certain cost function. This cost function attends to economical, technical, and environmental factors. It is relevant to note that this is a generic microgrid, being the proposed method equally valid to any other microgrid scheme.

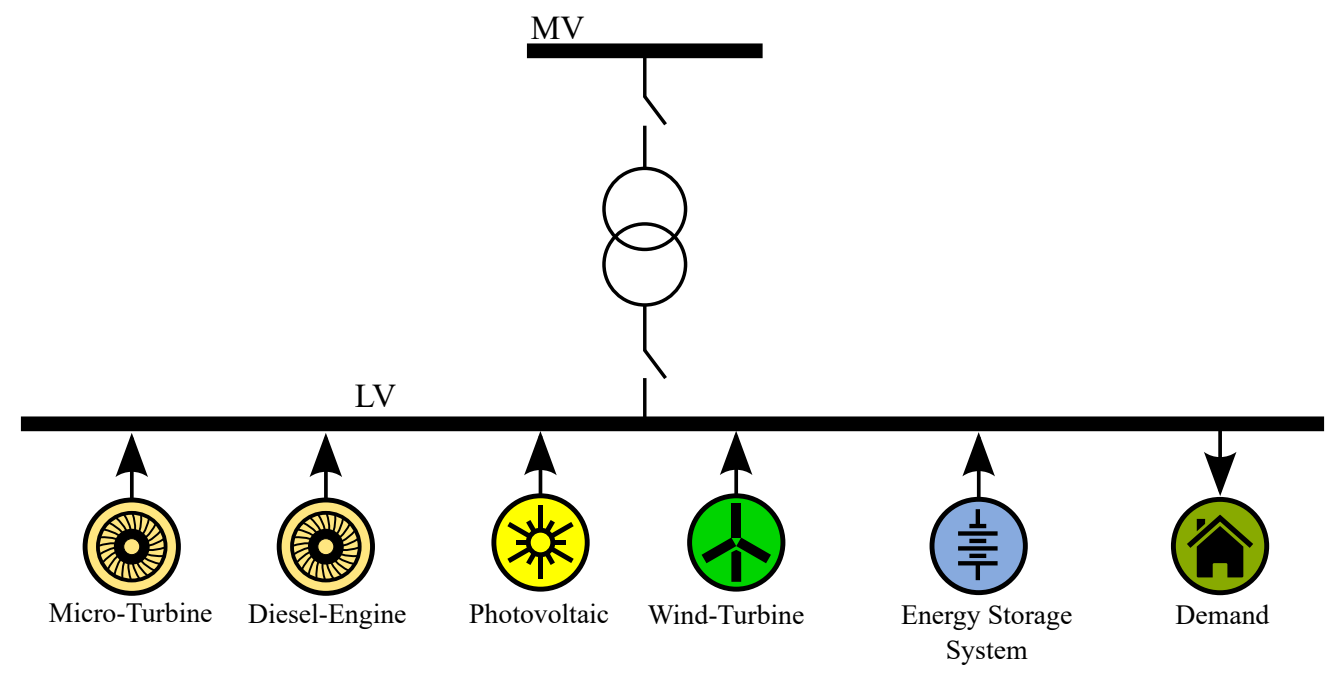

Figure 1. Structure of the microgrid considered. 


\section{System Modeling}

The upcoming sections deal with the model taken into consideration for the generation schedule problem. All models presented in this section have been used widely, however, in practice, their accuracy can vary substantially. Nevertheless, since the paper presents a robust solution to the UC and ED problems, when uncertainties in the demand forecasting are considered, greater uncertainties can be established in order to take into account other model inaccuracies.

\subsection{Demand Forecasting}

One important issue that has a significant impact on the solution of the problem is the accuracy of the considered data. The energy demanded in the microgrid implies the effective utilization of the energy resources to ensure reliability level in the energy supply. Nevertheless, the demand in a microgrid is usually estimated based on the habits of the customers and data records. This demand forecasting can fall into inaccuracy when no information about the grid is known or new entities connect to the network.

In this paper, we consider an estimated curve of demand that is affected by a final error. This error is considered to follow a normal distribution at every time instant $t, \mathcal{N}\left(\mu_{t}, \sigma_{t}\right)$, whose average, $\mu_{t}$, is considered zero and the standard deviation, $\sigma_{t}$, varies according to the level of reliability required to the problem resolution. In that way, if we consider a high value of $\sigma_{t}$, the uncertainty in the demand forecasting will be greater and a more robust solution must be contributed to guarantee the electrical supply under normal operation.

Let $\tilde{P}_{D M, t}$ and $P_{D M, t}$ be the estimation and the real power demanded from the microgrid at time $t$, respectively, and let $e_{D M, t}$ be the estimation error of the demand forecasting at time $t$. Thus, it is clear that

$$
\tilde{P}_{D M, t}+e_{D M, t}=P_{D M, t}, \quad \forall t,
$$

with $e_{D M, t} \sim \mathcal{N}\left(0, \sigma_{t}\right)$.

It is worth pointing out that, for the normal distribution $\mathcal{N}\left(\mu_{t}, \sigma_{t}\right)$, the values less than one standard deviation, $\sigma_{t}$, away from the mean, $\mu_{t}$, account for $68.27 \%$ of the set, while two and three standard deviation away from the mean account for $95.45 \%$ and $99.73 \%$ of the set, respectively [44]. Thus, if the solution of the problem guarantees the supply of a power demanded $P_{D M, t} \in\left[\tilde{P}_{D M, t}-\right.$ $\left.3 \sigma_{t}, \tilde{P}_{D M, t}+3 \sigma_{t}\right]$, then a reliable solution is achieved with a high confidence interval. To obtain a good estimation of the demand forecasting uncertainties, an ARMA (Auto Regressive Moving Average) model was trained with two years of estimation data.

\subsection{Photo-Voltaic Generator}

A PV generator consists of a set of PV panels connected among them that provide power to one or more solar inverters. These inverters transform the DC power received into AC power that is finally consumed in the grid. The power produced by a PV plant depends on many factors, such as the solar irradiation, the ambient and solar cell temperatures, and the modules degradation. Furthermore, the power generated by these kind of systems is directly related with the operational point of the solar modules. This operational point is fixed by the power inverter by using a Maximum Power Point Tracking (MPPT). In that way, the system will always operate supplying the maximum amount of power available in the resources.

In this paper, we consider the simplified model presented in [45] that is shown next:

$$
P_{P V, t}=P_{S T C} \frac{n \cdot E_{M, t}}{E_{S T C}}\left[1+k\left(T_{M, t}-T_{S T C}\right)\right], \quad \forall t,
$$

where $P_{P V, t}$ is the output power of the PV plant at time $t, E_{M, t}$ is the solar irradiance at $t$ and $P_{S T C}$, $E_{S T C}$ and $T_{S T C}$ are the the maximum power, the irradiance and the temperature under Standard Test Conditions (STC), respectively. Those values correspond to a cell temperature of $25{ }^{\circ} \mathrm{C}$ and 
an irradiance of $1000 \mathrm{~W} / \mathrm{m}^{2}$ with an air mass 1.5. Finally, $n$ denotes the number of PV panels, $k$ is the power temperature coefficient $\left({ }^{\circ} \mathrm{C}\right)$ and $T_{M, t}$ is the temperature of the module at time $t$, which can be calculated as $T_{M, t}=T_{a m b}+\varepsilon_{P V} \frac{E_{M, T}}{E_{S T C}}$, where $T_{a m b}$ is the ambient temperature $\left({ }^{\circ} \mathrm{C}\right)$ and $\varepsilon_{P V}$ is a constant module provided by the manufacturer.

\subsection{Wind Turbine}

There exist many publications dealing with the modeling and simulation of wind turbines [46,47]. Most of these models have a great level of detail and are used below to perform stability analysis (see, for instance, [48]). For our study, we consider the sequel simplified model of the generator:

$$
P_{W T, t}=\left\{\begin{array}{clc}
0 & \text { for } & v<v_{c i}, \\
P_{r} \frac{v^{3}-v_{c i}^{3}}{v_{r}^{3}-v_{c i}^{3}} & \text { for } & v_{c i}<v<v_{r}, \quad \\
P_{r} & \text { for } & v_{r}<v<v_{c o},
\end{array} \quad \forall t,\right.
$$

where $P_{r}$ and $v_{r}$ represent the rated power $(k W)$ and wind speed $(\mathrm{m} / \mathrm{s})$, respectively, and $v_{c i}, v_{c o}$ and $v$ are the cut-in, cut-out and actual wind speed, respectively. For a more detailed information about the model, reader is referred to [49].

\subsection{Diesel Engine}

Nowadays, diesel engines are the most common type of microgrid generator technology in use. The operation cost of a power system can be expressed as a function of its real power output and it is usually modeled as a quadratic polynomial with the shape:

$$
C_{D E, t}=d_{D E}+e_{D E} P_{D E, t}+f_{D E} P_{D E, t}^{2}, \quad \forall t,
$$

where $C_{D E, t}$ is the total fuel cost $(€ / \mathrm{h})$ at time $t, P_{D E, t}$ is the power output at time $t$ and $d_{D E}(e / \mathrm{h})$, $e_{D E}(e / \mathrm{kWh})$ and $f_{D E}\left(e / \mathrm{kW}^{2} \mathrm{~h}\right)$ are parameters specified by the manufacturer [50], and are strongly conditioned by the type of engine, generator and fuel. Additionally, let $P_{D E}^{\min }$ and $P_{D E}^{\max }$ be the minimum and maximum power that the DE can supply at every time instant. Then,

$$
P_{D E}^{\min } \leq P_{D E, t} \leq P_{D E}^{\max }
$$

\subsection{Microturbine}

The MT model is similar to the DE model. Nevertheless, in this case, the operation cost curve parameters are adopted to model the performance and efficiency of a MT unit [51]. Thus, the operation cost can be expressed as:

$$
C_{M T, t}=d_{M T}+e_{M T} P_{D E, t}+f_{M T} P_{M T, t \prime}^{2} \quad \forall t,
$$

where $C_{M T, t}$ is the total gas cost $(€ / \mathrm{h})$ at time $t, P_{M T, t}$ is the power output at time $t$ and $d_{M T}(€ / \mathrm{h})$, $e_{M T}(€ / \mathrm{Kwh})$ and $f_{M T}\left(€ / \mathrm{Kw}^{2} \mathrm{~h}\right)$ are parameters specified by the manufacturer [50,51], and depend on the type of turbine, generator and gas. The operation cost curves of the DE and MT in function of the power given will be presented in subsequent sections. Analogously with the DE, the power generated by the MT is physically limited:

$$
P_{M T}^{\min } \leq P_{M T, t} \leq P_{M T}^{\max }
$$

where $P_{M T}^{\min }$ and $P_{M T}^{\max }$ are the minimum and maximum power that the DE can supply at every time instant. 


\subsection{Spinning Reserve}

The term spinning reserve is widely used in the literature [52,53]. Nevertheless, this service can be defined in different ways leading to confusion. We define this concept as it is done in [54]: the spinning reserve is the unused capacity that can be activated on decision of the system operator and is provided by devices, which are synchronized to the network and able to change the active power.

Note that this capacity is only found in the dispatchable units, as they are the only units that can be used on demand. Thus, we denote $R_{D E, t}$ and $R_{M T, t}$ the spinning reserve of the DE and the MT at time instant $t$, respectively. These parameters are mathematically defined next:

$$
R_{D E, t}=P_{D E}^{\max }-P_{D E, t}, \quad R_{M T, t}=P_{M T}^{\max }-P_{M T, t}
$$

\subsection{Energy Storage System}

Batteries are electrochemical devices that store energy from other AC or DC sources for later use [51]. The battery allows the microgrid to cover demand peaks and to store excesses of generation due to its quick response. We consider a set of batteries that conforms an ESS. An ESS is particularized by three parameters:

1. The capacity is the amount of electric charge that the ESS can deliver at the rated voltage.

2. The maximum charge rate is a measure of the rate at which a battery can be charged.

3. The maximum discharge rate is a measure of the rate at which a battery can be discharged.

Let $P_{E S S, t}$ be the power demanded or supplied by the ESS at time $t$. Note that the value of $P_{E S S, t}$ can be both positive or negative. If $P_{E S S, t}>0$, the ESS is supplying power to the microgrid, while, when $P_{E S S, t}<0$, the ESS is charging and acting as a consumer. Attending to the charging/discharging rate, both parameters are bounded due to physical limitations:

$$
\begin{aligned}
& P_{E S S, t} \leq P_{E S S}^{\max }, \\
& P_{E S S, t} \geq P_{E S S}^{\min } .
\end{aligned}
$$

Let $S O C_{t}$ be the State of Charge of the ESS at every time $t$. According to the energy stored in the battery, the $S O C_{t}$ will take different values. Two constraints must be taken into consideration when dealing with the state of charge of the ESS. The $S O C_{t}$ must lay in a band bounded by the capacity of the battery and the minimum admissible state of charge. That is:

$$
S O C^{\min } \leq S O C_{t} \leq S O C^{\max }, \quad \forall t,
$$

where $S O C^{\min }$ and $S O C^{\max }$ are the lower and upper boundaries for the $S O C_{t}$. Additionally, it is important to point out that the battery is a dynamical system, that is, the state of charge of the ESS depends on the previous power flows through its terminals. Thus,

$$
S O C_{t+1}=S O C_{t}-\left\{\begin{array}{ccc}
P_{E S S, t} \Delta t \eta_{c} & \text { for } & P_{E S S, t}<0 \\
\frac{P_{E S S, t} \Delta t}{\eta_{d}} & \text { for } & P_{E S S, t}>0
\end{array}, \quad \forall t,\right.
$$

where $\eta_{c}$ and $\eta_{d}$ are, respectively, the charging and discharging efficiency and $\Delta t$ is the time between samples. Recall that, when $P_{E S S, t}>0$ and ESS is discharging, the $S O C_{t+1}$ decreases. Conversely, when $P_{E S S, t}<0$ and ESS is charging, the $S O C_{t+1}$ increases its value.

\subsection{Power Balance}

In contrast with other approaches to the same problem, similar to Nemati et al. [13], we consider an isolated microgrid, i.e., it lacks connection to the distribution network. This fact implies that the power demanded by the customers must be locally supplied by the distributed energy resources presented in the microgrid. Thus, the power balance must be fulfilled at every time interval $t$, that is 


$$
P_{D M, t}=P_{P V, t}+P_{W T, t}+P_{D E, t}+P_{M T, t}+P_{E S S, t}, \quad \forall t .
$$

It is important to point out that, when considering uncertainties in the demand forecasting, the estimation error must be taken into account in the problem in order to guarantee the reliability of the solution provided. This reliability is met with the well-sizing of the spinning reserve. Thus, the following constraint must be added to the problem:

$$
R_{D E, t}+R_{M T, t} \geq n_{r} \sigma_{t}
$$

where $\sigma_{t}$ is the standard deviation of the estimation error and $n_{r}$ is a scalar parameter that, once fixed, establishes the reliability degree of the solution adopted. A deeper study of this issue is presented in Section 5.3.

\subsection{Other Operation Costs}

In addition to all the costs aforementioned, the operation of the microgrid introduces extra costs associated to maintenance, start-up and emissions costs.

1. Maintenance cost: The operating and maintenance cost of each dispatchable generating unit is assumed to be proportional to the power production [51,55]:

$$
\begin{aligned}
& C_{O M_{D E}}=k_{O M_{D E}} \sum_{\forall t} P_{D E, t} \triangle t, \quad \forall t, \\
& C_{O M_{M T}}=k_{O M_{M T}} \sum_{\forall t} P_{M T, t} \triangle t, \quad \forall t,
\end{aligned}
$$

where $K_{O M_{D E}}(€ / \mathrm{kW} \mathrm{h})$ and $K_{O M_{M T}}(€ / \mathrm{kW} \mathrm{h})$ are the maintenance costs of DE and MT, respectively.

2. Start-up cost: The generator start-up cost depends on the time at which the unit has been off prior to start up [56]. Thus, the start-up cost at any given time can be estimated as [57]:

$$
\begin{aligned}
& C_{S U_{D E}}=a_{D E}+b_{D E}\left[1-\exp \left(\frac{-T_{D E, O F F}}{c_{D E}}\right)\right], \\
& C_{S U_{M T}}=a_{M T}+b_{M T}\left[1-\exp \left(\frac{-T_{M T, O F F}}{c_{M T}}\right)\right],
\end{aligned}
$$

where $a_{D E}$ and $a_{M T}(€)$ are the hot start-up costs, $b_{D E}$ and $b_{M T}(€)$ are the cold start-up costs, $c_{D E}$ and $c_{M T}(€)$ are the unit cooling time constant and $T_{D E, O F F}$ and $T_{M T, O F F}$ represent the time that each unit has been off (for a more detailed explanation about this expression, the reader is referred to [57]).

3. Emission costs: The cost of environmental externalities (production of pollutants $\mathrm{CO}_{2}, \mathrm{SO}_{2}$ or $\mathrm{NO}_{x}$ ) is assumed to be a linear function of energy generation [13]:

$$
\begin{aligned}
C E M_{D E} & =\sum_{k=1}^{N e m} \sum_{\forall t} \alpha_{k} \beta_{D E, k} P_{D E, t} \triangle t, \quad \forall t, \\
C E M_{M T} & =\sum_{k=1}^{N e m} \sum_{\forall t} \alpha_{k} \beta_{M T, k} P_{M T, t} \triangle t, \quad \forall t,
\end{aligned}
$$

where Nem is the number of emission types, $\alpha_{k}$ is externality costs of emission type $k(€ / \mathrm{kg})$, and $\beta_{D E, k}$ and $\beta_{M T, k}$ are the emission factors of the dispatchable generating units and the emission type $k(\mathrm{~kg} / \mathrm{kWh})$. 


\section{Microgrid Operation Modes}

As introduced in the previous sections, there exist a high number of limitations and cost parameters to take into consideration when scheduling the power generation in a microgrid. In this section, several operation modes are presented. In each of them, a certain operation criterion is followed to optimize a particular cost function.

\subsection{Cost-Effective Operation Mode}

This operation mode is focused on the reduction of the overall cost of the microgrid. Thus, the cost function considers the sequel parameters:

- $\quad$ cost of operation and maintenance of the dispatchable units (DE and MT);

- $\quad$ start-up costs of the dispatchable units (DE and MT); and

- fuel and gas costs of the DE and MT, respectively.

The cost function is mathematically defined as follows:

$$
C F_{e c}=C_{O M_{D E}}+C_{O M_{M T}}+C_{S U_{D E}}+C_{S U_{M T}}+\sum_{\forall t}\left(C_{D E, t}+C_{M T, t}\right)
$$

\subsection{Eco-Mode Operation}

In this operation mode, the main aim is to guarantee the electric supply in the microgrid, ensuring a minimization of the detrimental emissions that are the main cause of climate change. Climate change is expected to have unprecedented implications on where people can settle, grow food, build cities, and rely on functioning ecosystems for the services they provide. In many places, temperature changes and sea-level rise are already putting ecosystems under stress and affecting human well-being. This fact has induced many countries to set up policies to fight against climate change. At European level, a comprehensive package of policy measures to reduce greenhouse gas emissions has been initiated through the European Climate Change Program (ECCP).

Thus, based on the models defined in previous sections, the eco-cost function can be written as:

$$
C F_{e f}=C E M_{D E}+C E M_{M T}
$$

where it is important to note that the rest of elements does not imply any cost.

\subsection{Robust Operation Mode}

As mentioned in Section 4, this paper considers uncertainties in the demand forecasting. This issue becomes a problem when trying to operate the microgrid in isolated mode, i.e., disconnected from the main grid. Thus, it is reasonable to consider a complementary mode of operation that guarantees the reliability of the studied microgrid.

The consideration of these uncertainties in the problem is what is nowadays known as Stochastic Unit Commitment (SUC) problem. This problem requires a more robust solution, which is able to deal with the uncertainties presented in the proposed formulation. In this paper, we consider that the estimation error of the demand forecasting follows a normal distribution with mean zero and standard deviation at time $t, \sigma_{t}$, as exposed in Section 4.1. The solution proposed consists in designing the spinning reserves of the dispatchable units in such a way that the reserve power can meet the demand estimation error for several levels of reliability according to the value of $n_{r}$ introduced in Equation (14) (Figure 2 shows the reliability level for values of $n_{r}=1, n_{r}=2$ and $n_{r}=3$ ). 


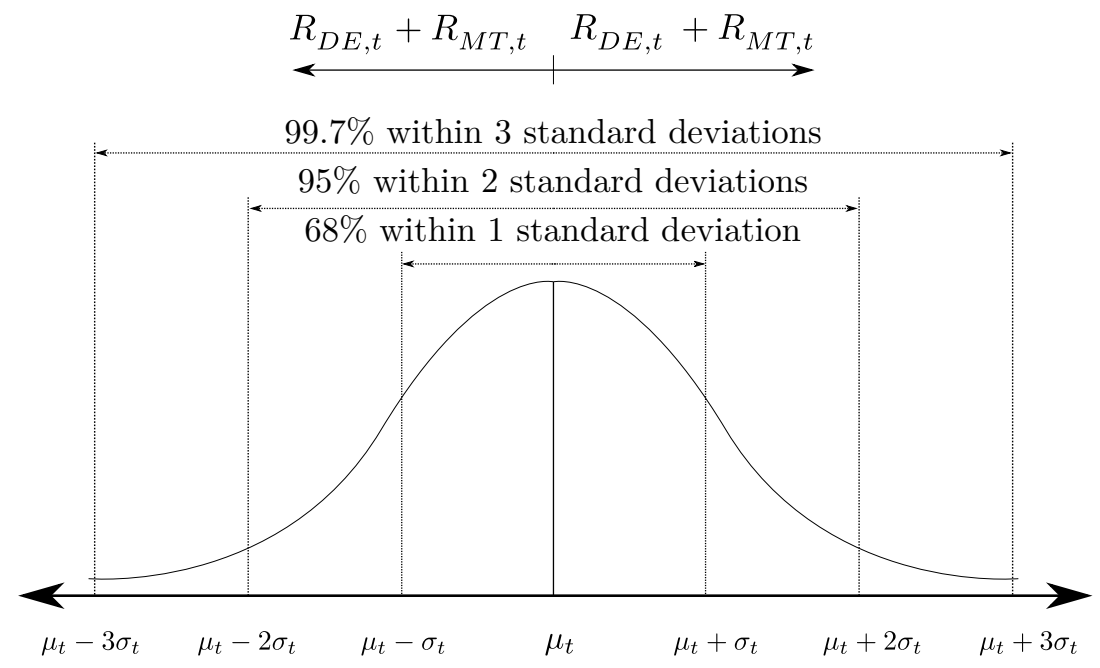

Figure 2. Normal distribution of the demand estimation error.

This mode of operation is complementary to the ones described above since it includes additional constraints to the problem, not changing the cost functions in Equations (21) and (22). In this mode, more expensive solutions are expected to be obtained, given the severe constraints related to robustness that they must fulfill.

\section{Evolutionary Computational Approach}

Genetic algorithms (GAs) are meta-heuristic algorithms that have been widely used for solving complex engineering problems $[58,59]$. GAs are based on the Darwinian theory of biological evolution. The basic idea is to have a set of potential solutions, called the population, which evolves over a number of generations by using genetic operators such as selection, crossover, and mutation [60]. The potential solutions encode in a chromosome-like structure the designing variables. This structure is called an individual and each designing variable represents a gene. In this work, the designing variables are the power supplied/consumed by MT, DE, and ESS since they are the elements of the considered microgrid that have higher impact on the operational cost of the microgrid (see Figure 1). RES, such as PV and WT, will always contribute to the demanded power as long as the solar and wind conditions are favorable, since their maintenance and operational costs can be neglected with respect to the DE and MT generators. Furthermore, since the power balance at each time instant should be fulfilled, whether the ESS supply or consume power at each time interval $t$, is determined by the power balance between the demand and the generation of the rest of elements of the microgrid.

\subsection{Genetic Algorithm Implementation}

There are many possible implementations of single-objective GAs [61]. In the proposed approach, a mupluslambda variant is used [62]. Algorithm 1 shows the implemented approach. The algorithm begins with a random initial population $P_{i}$, which is evaluated. It is important that the individuals that form the initial population are valid. This aspect is relevant in problems with multiple constrains as the one described in this paper (see Section 4). Then, offspring $\mu$ is created by using crossover and mutation operations. $p_{c x}$ and $p_{m u t}$ refer to the crossover and mutation probabilities, respectively. Both $p_{c x}$ and $p_{m u t}$ are hyper-parameters of the algorithm that should be selected carefully to guarantee a good converge of the GA. Next, the offspring is evaluated, and the new population $\lambda$ is selected from the offspring generated and the previous population $P_{g}$. This approach guarantees a good level of elitism since parents and offspring compete each other to be selected for the next generation [62]. After completion, the algorithm returns the best individual obtained throughout the generations. 


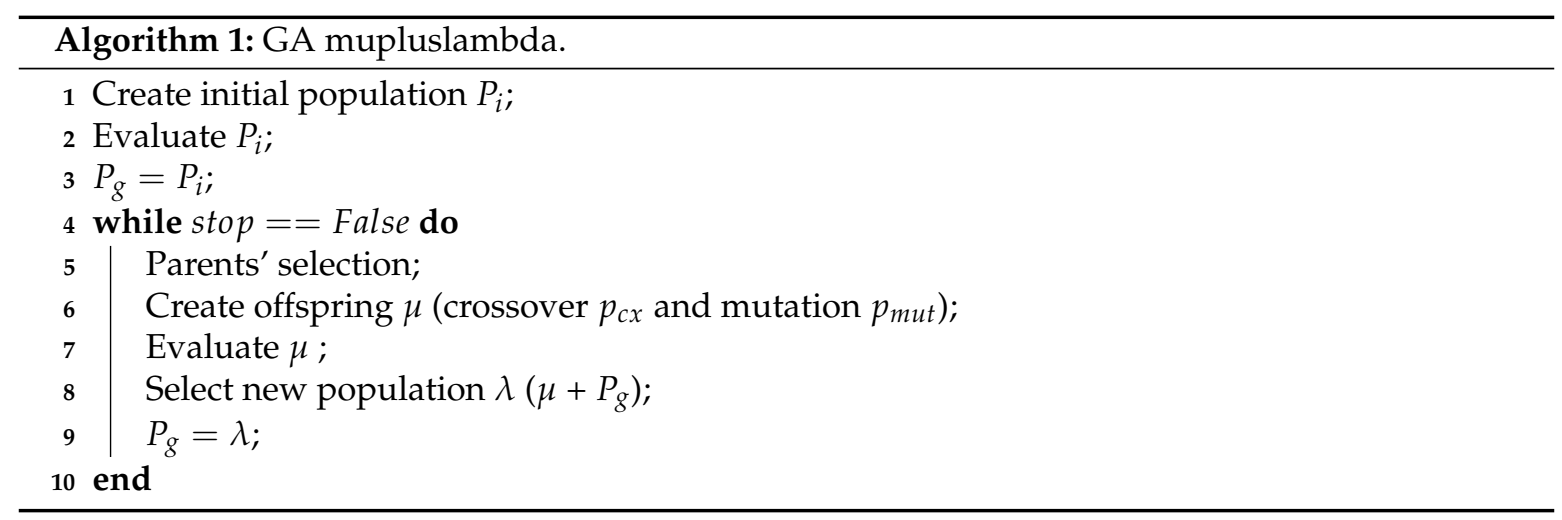

\subsubsection{Individual Representation}

Each individual represents a list containing the power supplied by both MT and DE for a period of $24 \mathrm{~h}$, using intervals of $1 \mathrm{~h}$. Therefore, the size of each individual is 48 genes. It is worth recalling that the power supplied or consumed by the ESS can be derived from the power balance, and the renewable sources will always contribute to the demanded power as long as the climate conditions are suitable. Figure 3 shows the encoding used to represents the individuals of the GA.

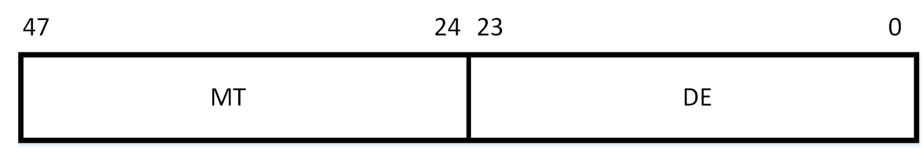

Figure 3. Individual representation.

\subsubsection{Fitness Function}

The fitness function used depends on the operation mode of the microgrid. Three operation modes have been defined in Section 5 that use a single-objective fitness function, such as cost-effective, eco-mode, and robust operation modes.

In the case of cost-effective mode, the objective function is defined as:

$$
\left\{\begin{array}{cl}
\text { if solution valid } & F=\text { Equation (21), } \\
\text { else } & F=\infty, \text { according to Equations (5), (7), (9), (10), (11), (12), and (13). }
\end{array}\right.
$$

In the case of eco-mode, the objective function used is:

$$
\left\{\begin{array}{cl}
\text { if solution valid } & F=\text { Equation (22), } \\
\text { else } & F=\infty, \text { according to Equations (5), (7), (9), (10), (11), (12), and (13). }
\end{array}\right.
$$

In the case of robust mode, the fitness function used is:

$$
\left\{\begin{array}{cl}
\text { if solution valid } & F=\text { Equation (22), } \\
\text { else } & F=\infty, \text { according to Equations (5), (7), (8), (9), (10), (11), (12), (13), and (14). }
\end{array}\right.
$$

It is important to highlight that, in the three fitness functions in Equations (23)-(25), the death penalty is used to penalize invalid solutions. Therefore, these invalid solutions do not participate in the genetic operations since they are not chosen by the selection scheme.

\subsubsection{Genetic Operators}

Tournament selection mechanism is used since it provides suitable results [61]. In each tournament, a number of individuals are randomly selected, which compete each other to be chosen 
as a parent; the best one is then selected as one of the parents to be used in crossover and mutation operations [61]. A tournament size of three has been demonstrated to be suitable for the majority of problems. Regarding the crossover operation, two schemes have been evaluated: two-point and Simulated Binary Crossover (SBX) [63] methods. The two-point crossover consists of swapping the genetic information of two parents using two points as the indexes of the genetic exchange. SBX is a method to simulate one point crossover for continuous variables. An eta hyper-parameter is used to determine the similarity among selected parents and children. A high eta value will produce children resembling their parents, while a small eta value will produce much more different solutions. The mutation scheme used is a tailored Gaussian mutation algorithm, where each variable can change according to a Gaussian distribution with mean $\mu$ and standard deviation $\sigma$. The variability of a given gene after mutation depends on the value of $\sigma$.

\section{Simulation Results}

In this section the application of the proposed evolutionary approach is discussed. For this, a particularization of the microgrid presented in Section 3 is first presented, being the GA applied to three different case-studies. Lastly, the results are shown together with the values of the cost functions for the different operation modes. A simulation framework was developed in Python using DEAP library [64]. The simulator is available in [65].

\subsection{Example scenario settings}

The proposed example scenario consisted in solving the $24 \mathrm{~h}$ short-term operation scheduling of the microgrid introduced in Section 3. A sample time of $1 \mathrm{~h}$ was considered in all the simulations, however, the consideration of a shorter sampling is also possible. For this microgrid, two dispatchable units (DE and MT), two renewable resources (PV and WT) and an ESS were considered. The configuration parameters of each of the dispatchable generators together with their operation, start-up and maintenance costs coefficients are exposed in Table 2.

Table 2. Characteristic parameters of each of the generators considered in the microgrid.

\begin{tabular}{llllll}
\hline $\boldsymbol{i}$ & $\boldsymbol{P}_{\boldsymbol{i}}^{\min }(\mathrm{kW})$ & $\boldsymbol{P}_{\boldsymbol{i}}^{\max }(\mathbf{k W})$ & $\boldsymbol{d}_{\boldsymbol{i}}(€ / \mathbf{h})$ & $\boldsymbol{e}_{\boldsymbol{i}}(€ / \mathbf{k W h})$ & $f_{\boldsymbol{i}}\left(€ / \mathbf{k W}^{2} \mathbf{h}\right)$ \\
\hline $\mathrm{DE}$ & 5 & 80 & 1.9250 & 0.2455 & 0.0012 \\
$\mathrm{MT}$ & 20 & 140 & 7.4344 & 0.2015 & 0.0002 \\
\hline $\boldsymbol{i}$ & $\boldsymbol{a}_{\boldsymbol{i}}(€)$ & $\boldsymbol{b}_{\boldsymbol{i}}(€)$ & $\boldsymbol{c}_{\boldsymbol{i}}(€)$ & $\boldsymbol{K}_{\boldsymbol{O} M_{i}}(€ / \mathbf{k W h})$ \\
\hline $\mathrm{DE}$ & 0.3 & 0.4 & 5.2 & 0.01258 & \\
$\mathrm{MT}$ & 0.4 & 0.28 & 7.1 & 0.00587 & \\
\hline
\end{tabular}

Furthermore, Figure 4 depicts the operation costs of the dispatchable units. Notice that the DE generation is cheaper for power values under $55 \mathrm{~kW}$. It is worth pointing out that the operation cost of DE is lower than MT when supplying low power. Nevertheless, DE is much more polluting than MT (see Table 3). Therefore, when the power given by the generator increases significantly, MT is the cheaper and lower-emissions solution. This fact makes cost the functions in Equations (21) and (22) have a minimum value in two different operation points.

In Figure 5, the start-up cost is depicted for both dispatchable units with respect to the time that the unit has been off. As can be observed, the cold start of MT has a higher cost than DE; nevertheless, after $5 \mathrm{~h}$ of not operation, MT is cheaper than reactivating.

The cost coefficients of Equations (19) and (20) are particularized for DE and MT in Table 3. We considered three emission types: $\mathrm{CO}_{2}, \mathrm{SO}_{2}$ and $\mathrm{NO}_{x}$. The ESS parameters are defined in Table 4. 


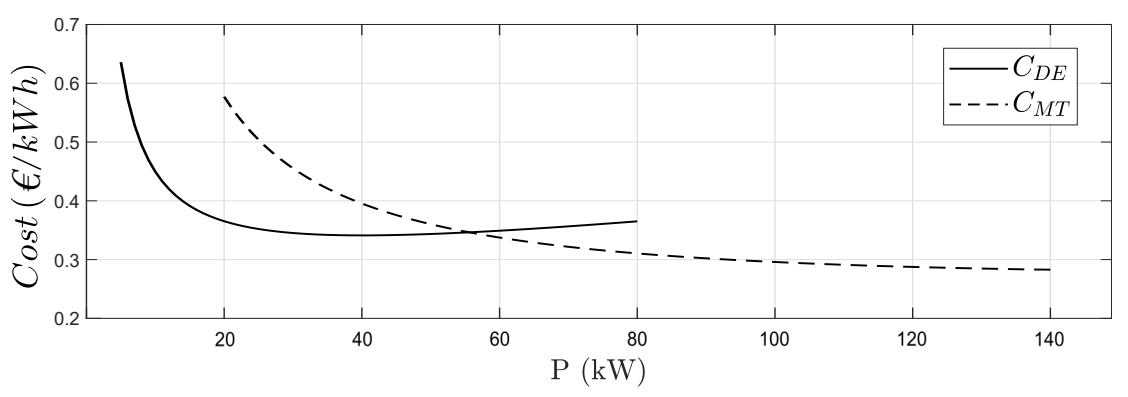

Figure 4. Cost of operation of the DE and MT in terms of the power supply.

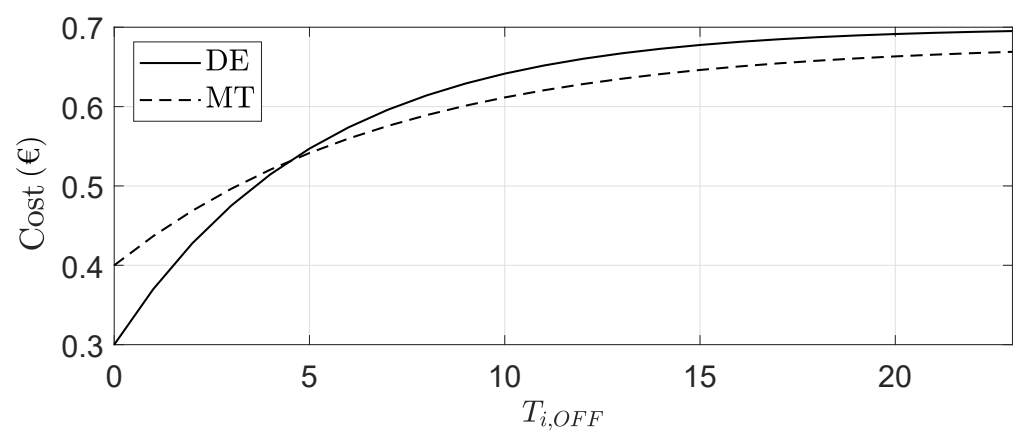

Figure 5. Start-up cost of the dispatchable units in terms of the time the unit has been off.

Table 3. Parameters of the externality costs and the emission factors of the main grid generation.

\begin{tabular}{llll}
\hline Emission Type & $\alpha_{k}$ & $\beta_{D E, k}$ & $\beta_{M T, k}$ \\
\hline $\mathrm{CO}_{2}$ & 0.0275 & 0.6495 & 0.7239 \\
$\mathrm{SO}_{2}$ & 1.9475 & 0.2059 & 0.0036 \\
$\mathrm{NO}_{x}$ & 8.2625 & 9.8883 & 0.1995 \\
\hline
\end{tabular}

Table 4. ESS characteristic parameters.

\begin{tabular}{llllll}
\hline$S_{S O C^{\min }(\mathbf{k W h})}$ & $S^{\operatorname{moC}}{ }^{\max }(\mathbf{k W h})$ & $\boldsymbol{P}_{E S S}^{\min }(\mathbf{k W})$ & $\boldsymbol{P}_{E S S}^{\max }(\mathbf{k W})$ & $\eta_{c}$ & $\eta_{d}$ \\
\hline 70 & 280 & -120 & 120 & 0.9 & 0.9
\end{tabular}

The power demanded and the renewable resources generation for the short $24 \mathrm{~h}$ period considered are known in the problem. These curves are shown in Figure 6. The problem was solved considering one datum per hour. It is important to highlight that this time can be reduced, implying the increment of the number of variables of the problem, but preserving the problem formulation made in this work.

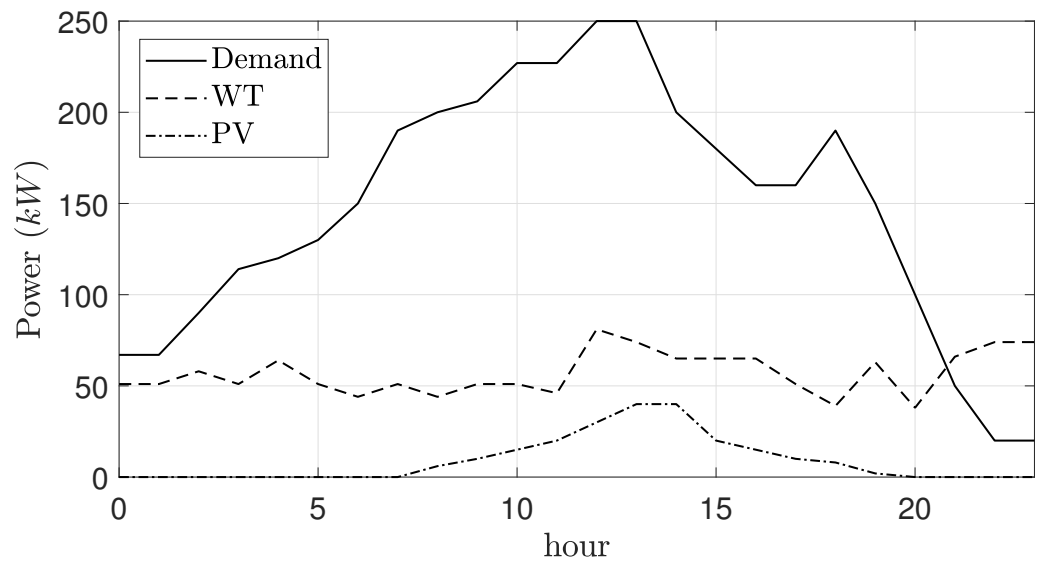

Figure 6. Power demanded and generated with renewable resources. 


\subsection{Genetic Algorithm Settings}

The design of the generation schedule of the microgrid was addressed by means of a GA, using the selection, crossover and mutation rules proposed in Section 6. Table 5 contains the main configuration parameters of the GA implementation. It can be noticed that the proposed GA qA tested under different configuration parameters in terms of crossover and mutation probabilities. In addition, for the SBX method, a detailed evaluation of the influence of the eta hyper-parameter Qa carried out.

Table 5. Parameters of the GA.

\begin{tabular}{ll}
\hline Parameter & Value \\
\hline$\lambda$ & 3000 \\
$\mu$ & 3000 \\
Individuals (multi-objective) & 3000 \\
Generations & 1000 \\
Selection & Tournament size $=3$ \\
Crossover & Two-point scheme and Binary Simulated $p_{c x}=[0.6,0.7,0.8]$. \\
Mutation & Gaussian $p_{m}=[0.4,0.3,0.2], \sigma=30$. \\
Number of trials & 30. \\
\hline
\end{tabular}

To show the convergence of the proposed GA, Figure 7 depicts the evolution of the individuals throughout the considered number of generations. It is shown that 1000 generations sufficed to guarantee convergence. Notice that the convergence of the algorithm could be affected by the value of $\sigma$ used in the mutation algorithm. Similar convergence degrees were obtained for the rest of case-studies with the same value of $\sigma$.

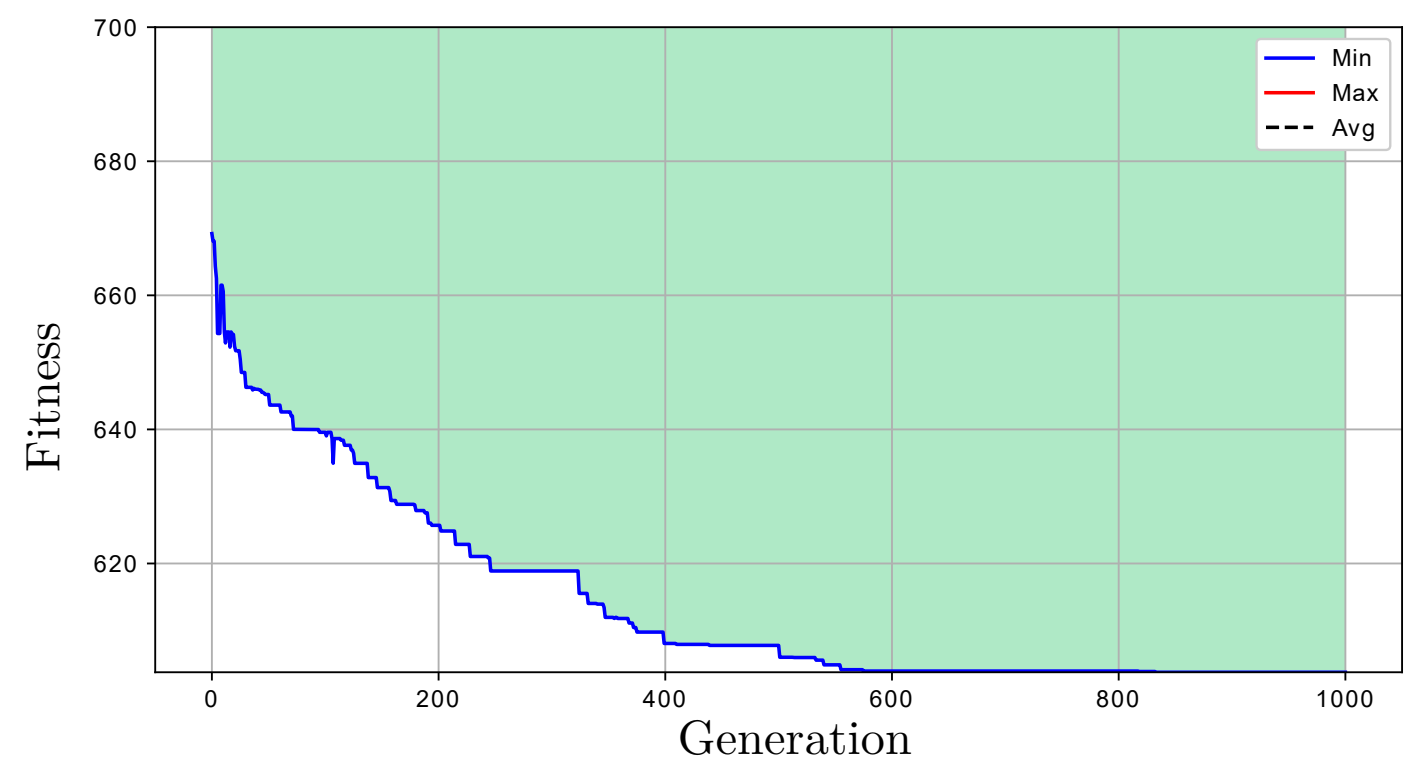

Figure 7. Evolution of the proposed GA, Case Study 1, two-point crossover, $p_{c}=0.6$ and $p_{m}=0.4$.

\subsection{Results for the Case Studies}

For this problem, three different scenarios are proposed, being the results listed below for each of them.

\subsubsection{Results for Case-Study 1: Cost-Effective Operation Mode}

This case study considered the resolution of the problem by minimizing the cost function in Equation (21). The best solutions obtained using the GA for this case-study are listed in Table 6 . In addition, in Figure 8, the best solution for the microgrid schedule is represented. 
Table 6. Case-study 1 results, expressed in $€$.

\begin{tabular}{|c|c|c|c|c|c|c|c|c|c|}
\hline \multicolumn{7}{|c|}{ SBX } & \multicolumn{3}{|c|}{ Two-Points } \\
\hline$P_{c x}$ & 0.6 & 0.7 & 0.8 & 0.6 & 0.7 & 0.8 & 0.6 & 0.7 & 0.8 \\
\hline$P_{m u t}$ & 0.4 & 0.3 & 0.2 & 0.4 & 0.3 & 0.2 & 0.4 & 0.3 & 0.2 \\
\hline eta & & 0.01 & & & 0.1 & & & - & \\
\hline $\operatorname{best}\left(C F_{e c}\right)$ & 592.637 & 595.718 & 594.888 & 593.630 & 594.013 & 592.637 & 595.173 & 597.070 & 595.863 \\
\hline mean $\left(C F_{e c}\right)$ & 602.995 & 603.793 & 606.089 & 603.370 & 604.439 & 606.066 & 603.146 & 604.071 & 605.758 \\
\hline$\sigma\left(C F_{e c}\right)$ & 5.725 & 3.769 & 5.930 & 4.884 & 4.065 & 6.863 & 4.513 & 4.553 & 6.237 \\
\hline$P_{c x}$ & 0.6 & 0.7 & 0.8 & 0.6 & 0.7 & 0.8 & & & \\
\hline$P_{\text {mut }}$ & 0.4 & 0.3 & 0.2 & 0.4 & 0.3 & 0.2 & & & \\
\hline eta & & 1 & & & 10 & & & & \\
\hline $\operatorname{best}\left(C F_{e c}\right)$ & 594.390 & 596.204 & 596.737 & 596.543 & 594.180 & 597.118 & & & \\
\hline mean $\left(C F_{e c}\right)$ & 604.762 & 604.467 & 608.589 & 605.210 & 604.773 & 609.207 & & & \\
\hline$\sigma\left(C F_{e c}\right)$ & 6.793 & 4.449 & 6.823 & 6.040 & 6.059 & 6.217 & & & \\
\hline
\end{tabular}

Observing Table 6, it can be seen that the proposed approach led to very similar solutions, reaching the best one under the SBX scheme with $P_{c x}=0.6, P_{m u t}=0.4$ and et $a=0.1$. Analyzing Figure 8 , it can be seen that MT, when it was connected to the microgrid, generated high amounts of power, which is in accordance with the operation costs shown in Figure 4. Analogously, DE always providws low power when connected, reducing in that way the operational costs.

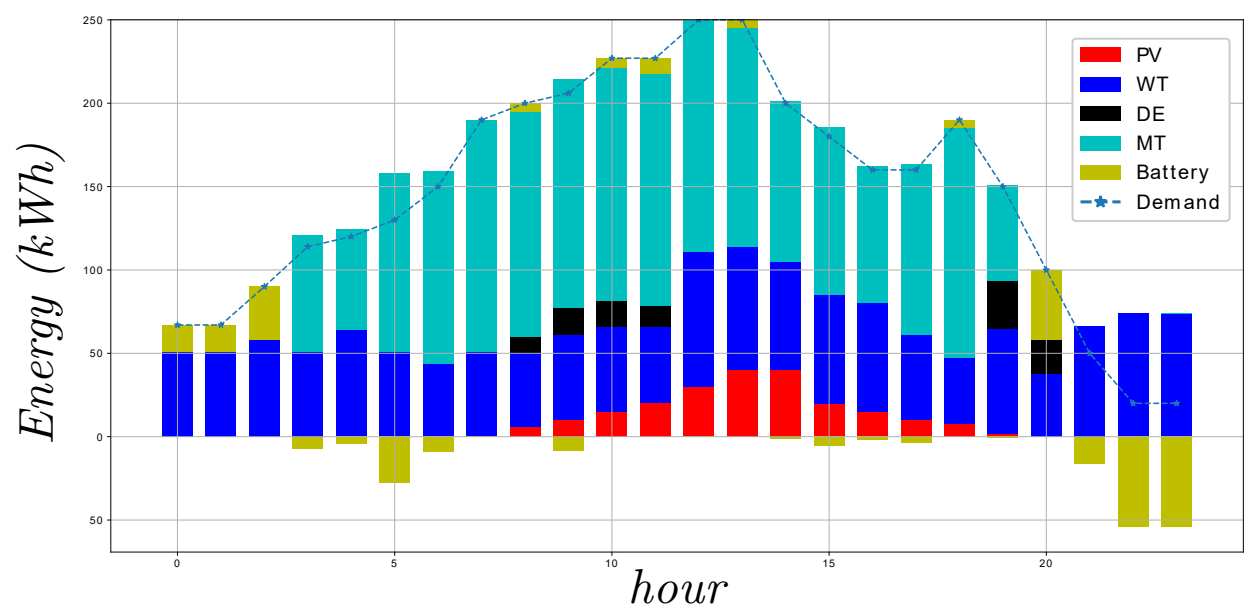

Figure 8. UC and ED solution for Case Study 1.

Finally, Figure 9 shows the evolution of the SOC and the power supplied or demanded by the ESS.

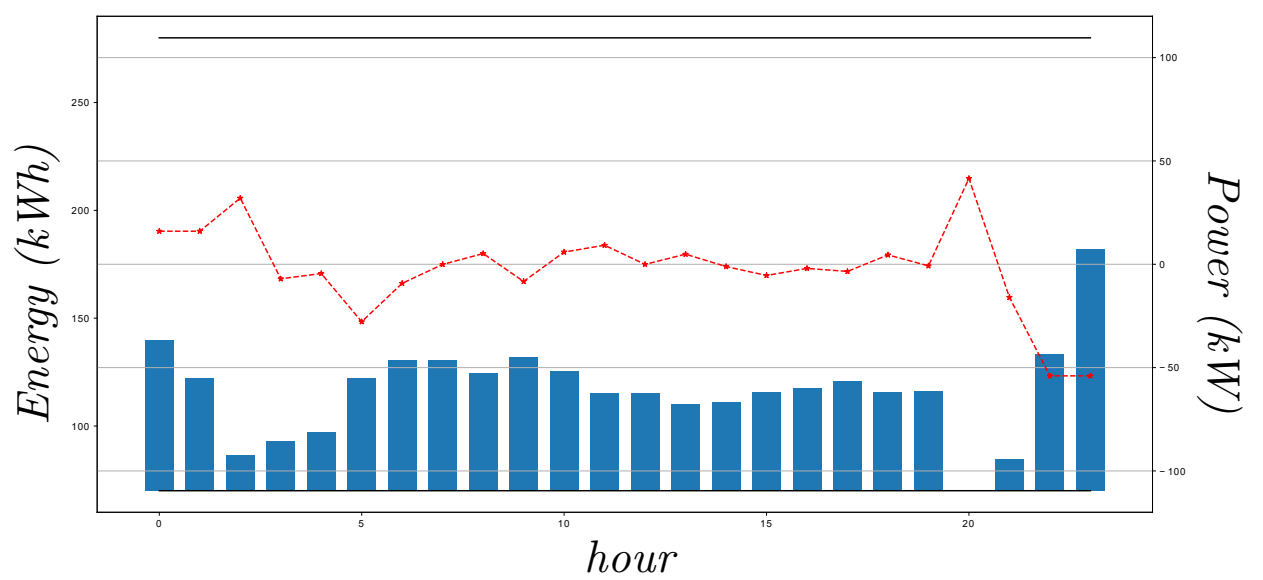

Figure 9. Battery management for Case Study 1 where the bars are the SOC at every time instant and the red dashed line is the power given or required by the battery. 


\subsubsection{Results for Case-Study 2: Eco-Mode Operation Mode}

This case study considered the resolution of the problem by minimizing the cost function in Equation (22). The best solution is depicted in Figure 10.

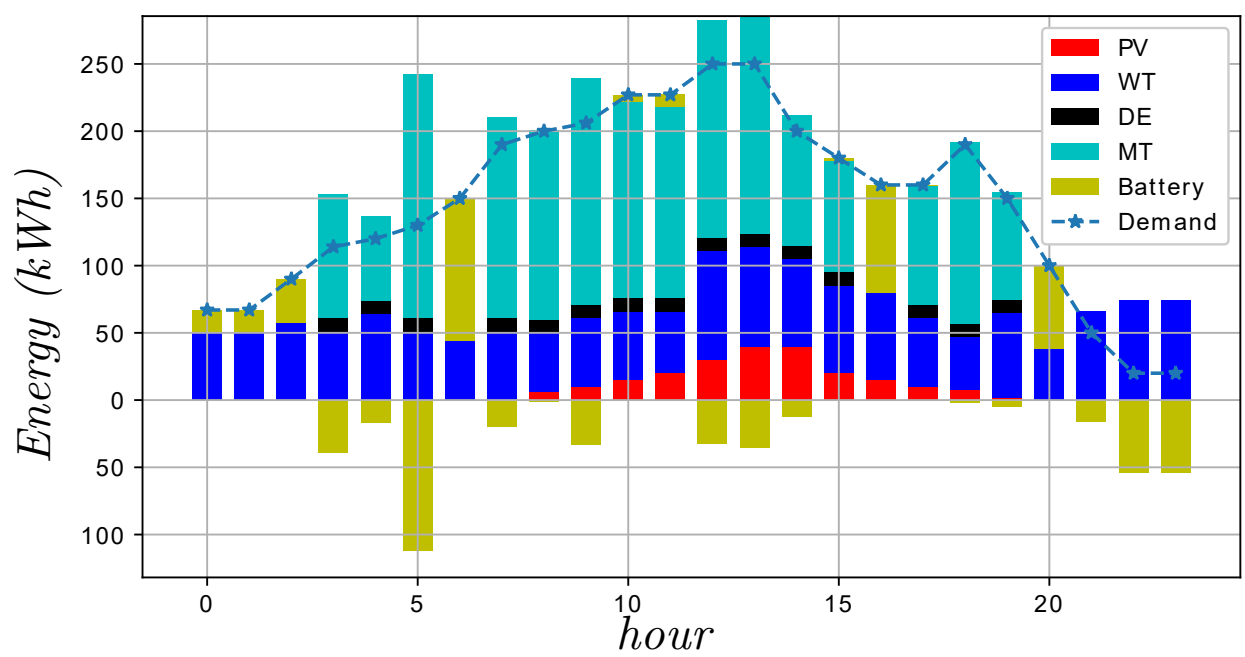

Figure 10. UC and ED solution for Case Study 2.

Different schemes were used to solve the GA problem. Table 7 shows the best solutions obtained. Note that this time the solution given by the SBX method with $P_{c x}=0.7, P_{m u t}=0.3$ and eta $=0.01$ was clearly better than the others. Furthermore, analyzing Figure 10, it can be seen that the DE generated less power in contrast with Case Study 1. This fact is a consequence of the high level of emissions produced by DE, as exposed in Table 3. Additionally, the EES was managed to disconnect the dispatchable units from the network at Hour 6, thus reducing the emissions in the microgrid.

Table 7. Case-study 2 results.

\begin{tabular}{|c|c|c|c|c|c|c|}
\hline \multicolumn{7}{|c|}{ SBX } \\
\hline$P_{c x}$ & 0.6 & 0.7 & 0.8 & 0.6 & 0.7 & 0.8 \\
\hline$P_{m u t}$ & 0.4 & 0.3 & 0.2 & 0.4 & 0.3 & 0.2 \\
\hline eta & & 0.01 & & & 0.1 & \\
\hline $\operatorname{best}\left(C F_{e c}\right)$ & $15,577.973$ & $15,511.697$ & $17,043.111$ & $15,525.373$ & $16,279.030$ & $15,497.908$ \\
\hline $\operatorname{mean}\left(C F_{e c}\right)$ & $17,586.300$ & $17,597.337$ & $18,034.655$ & $17,386.520$ & $17,593.823$ & $17,983.249$ \\
\hline$\sigma\left(C F_{e c}\right)$ & 775.725 & 954.216 & 771.705 & 714.173 & 635.349 & 635.349 \\
\hline$P_{c x}$ & 0.6 & 0.7 & 0.8 & 0.6 & 0.7 & 0.8 \\
\hline$P_{m u t}$ & 0.4 & 0.3 & 0.2 & 0.4 & 0.3 & 0.2 \\
\hline eta & & 1 & & & 10 & \\
\hline $\operatorname{best}\left(C F_{e c}\right)$ & $16,359.787$ & $16,395.022$ & $17,095.309$ & $15,580.220$ & $16,470.354$ & $17,324.083$ \\
\hline $\operatorname{mean}\left(C F_{e c}\right)$ & $17,517.452$ & $17,948.340$ & $17,925.474$ & $17,535.896$ & $17,857.965$ & $18,946.675$ \\
\hline$\sigma\left(C F_{e c}\right)$ & 805.524 & 694.802 & 524.494 & 765.017 & 662.151 & 892.987 \\
\hline \multicolumn{7}{|c|}{ Two-Points } \\
\hline$P_{c x}$ & 0.6 & 0.7 & 0.8 & & & \\
\hline$P_{m u t}$ & 0.4 & 0.3 & 0.2 & & & \\
\hline $\operatorname{best}\left(C F_{e c}\right)$ & $16,107.14$ & $17,904.88$ & $18,972.78$ & & & \\
\hline mean $\left(C F_{e c}\right)$ & $19,070.07$ & $19,878.66$ & $20,204.18$ & & & \\
\hline$\sigma\left(C F_{e c}\right)$ & 884.53 & 889.12 & 765.06 & & & \\
\hline
\end{tabular}

Figure 11 depicts the evolution of the SOC and the power supplied or demanded by the ESS. Note that, in this operation mode, the ES was used to reduce the power generated by the dispatchable units, unlike the cost-effective operation mode, whose aim was to minimize the cost of generation of the power units. 


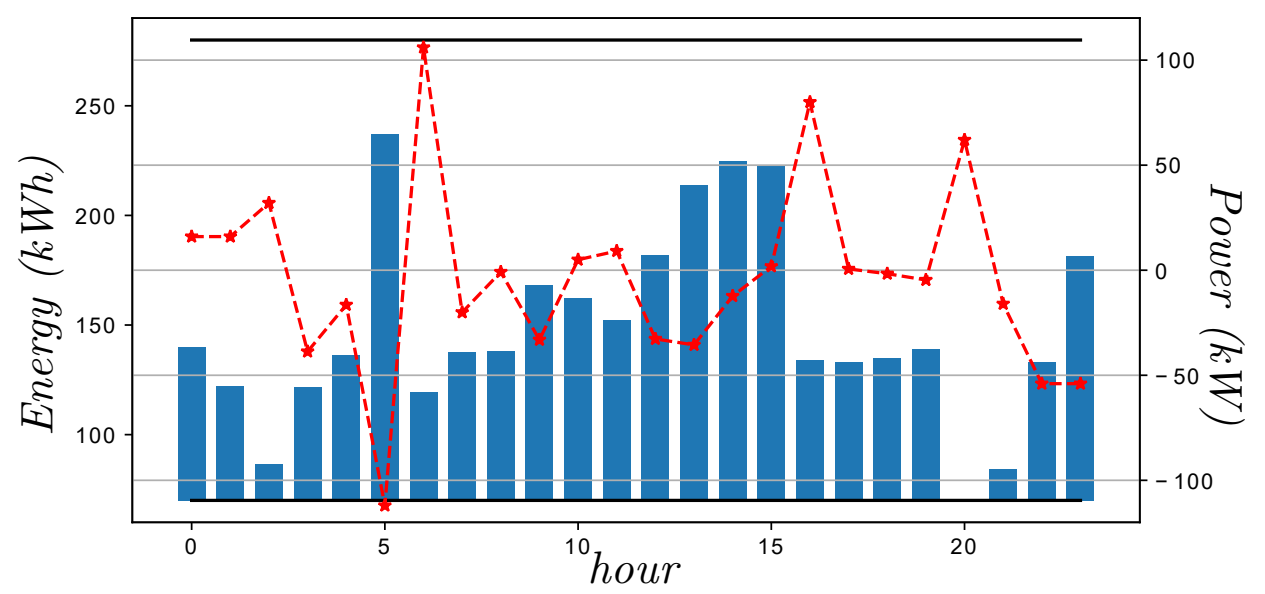

Figure 11. Battery management for Case Study 2 where the bars are the SOC at every time instant and the red dashed line is the power given or required by the battery.

\subsubsection{Results for Case-Study 3: Robust Operation Mode}

In this operation mode, an $n_{r}=3$ level of reliability was guaranteed. That is, the isolated operation of the microgrid was guaranteed in $99.7 \%$ of scenarios. The objective of the problem was the same as in the cost-effective operation mode, i.e. minimize the total operation and maintenance cost in the microgrid.

Table 8 shows the results of the GA for this case study. Note that, in contrast with Case Study 1 , the fitness function took higher values. This fact is a consequence of the reserve power, which must cover the possible deviation in the demand forecasting. As can be seen, the best solution was obtained for two-points, when the probabilities of crossover and mutation were, respectively, 0.8 and 0.2 .

Table 8. Case-study 3 results.

\begin{tabular}{c|ccc|ccc|ccc}
\hline \multicolumn{9}{c|}{ SBX } & \multicolumn{3}{c|}{ Two-Points } \\
\hline$P_{c x}$ & 0.6 & 0.7 & 0.8 & 0.6 & 0.7 & 0.8 & 0.6 & 0.7 & 0.8 \\
$P_{\text {mut }}$ & 0.4 & 0.3 & 0.2 & 0.4 & 0.3 & 0.2 & 0.4 & 0.3 & 0.2 \\
eta & & 0.01 & & & 0.1 & & & - & \\
\hline best $\left(C F_{e c}\right)$ & 610.804 & 613.942 & 615.287 & 612.770 & 611.888 & 612.615 & 613.579 & 614.154 & 610.792 \\
mean $\left(C F_{e c}\right)$ & 619.718 & 622.898 & 623.017 & 621.496 & 623.111 & 623.970 & 622.957 & 626.400 & 625.598 \\
$\sigma\left(C F_{e c}\right)$ & 4.017 & 4.198 & 5.130 & 4.921 & 4.604 & 5.563 & 4.301 & 5.578 & 6.468 \\
\hline$P_{c x}$ & 0.6 & 0.7 & 0.8 & 0.6 & 0.7 & 0.8 & & & \\
$P_{\text {mut }}$ & 0.4 & 0.3 & 0.2 & 0.4 & 0.3 & 0.2 & & & \\
eta & \multicolumn{1}{c|}{1} & 1 & & & 10 & & & & \\
\hline best $\left(C F_{e c}\right)$ & 613.722 & 613.636 & 615.364 & 613.483 & 614.783 & 617.842 & & & \\
mean $\left(C F_{e c}\right)$ & 621.852 & 625.127 & 625.648 & 621.520 & 624.422 & 627.119 & & & \\
$\sigma\left(C F_{e c}\right)$ & 4.399 & 5.210 & 5.573 & 5.122 & 5.773 & 6.373 & & & \\
\hline
\end{tabular}

Figures 12 and 13 show the generation schedule and the battery management for the predicted scenario, i.e., considering the possible uncertainties in the demand estimation. It is worth pointing out that, in this case, the battery allowed reducing the amount of energy provided by MT at every time step, increasing in this way the the spinning reserve of the microgrid. 


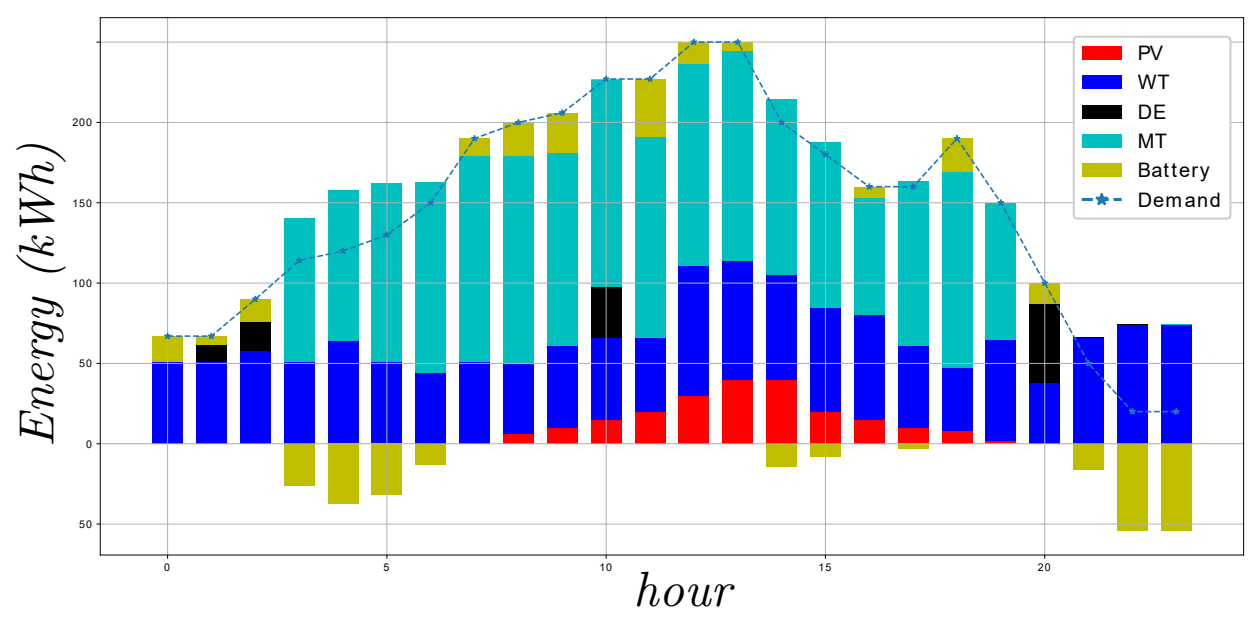

Figure 12. UC and ED solution for Case Study 3.

Finally, it is worth pointing out that the optimal solution in Case Study 2 (eco-mode operation mode) provided a value of 626.464 when it was evaluated by the cost function in Equation (21). Note that this value is not too far from the minimum. However, when replicating the same procedure with the optimal solutions for Case Studies 1 and 3 and evaluating by the cost function in Equation (22), it took the values of 4800.166 and 7518.955 , respectively, which are very far from the optimum.

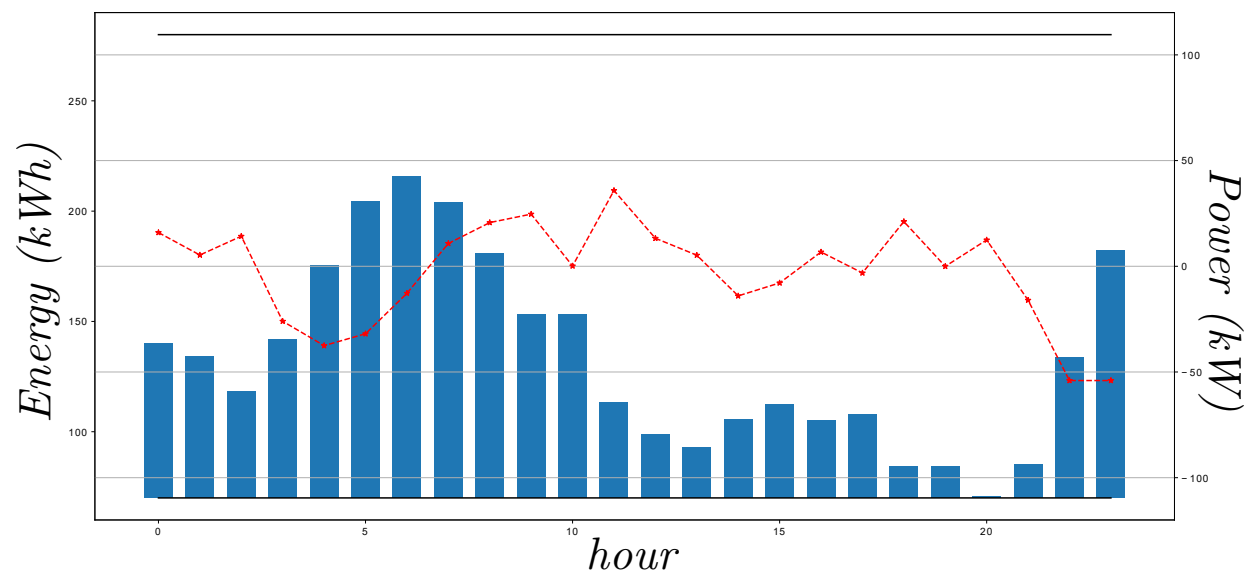

Figure 13. Battery management for Case Study 3 where the bars are the SOC at every time instant and the red dashed line is the power given or required by the battery.

\section{Conclusions and Further Work}

The present study was carried out for developing a GA to address the problem of finding the most suitable solution for the UC and ED problems in a microgrid. Based on the results, several conclusions can be formulated.

First, a detailed model of a microgrid was introduced to propose three case-studies (operation modes), which were addressed to validate the benefits of the GA to solve the combined problem of UC and ED. When the cost-effective operation mode was considered, the solution prioritized the use of DE instead of MT for low values of demanded power. However, when MT must be employed, it generated high amounts of power to have an appropriate power/cost ratio. With respect to the eco-mode operation mode, the microgrid minimized the use of DE due to its high levels of emissions, which are proportional to the power generated. In addition, the management of the battery system in both cases worked to reach the corresponding objectives, being an element of relevant importance to achieve this aim.

Regarding the limitations of the present work, the computational time required by the GA makes it low efficient to addressing the problem in real-time. In addition, despite the goodness of the obtained 
results, the absence of guarantee of finding global solutions must always be taken into account, as the algorithm might converge at local minimum. For this reason, an appropriate tuning of the GA parameters is necessary when modifying the problem scenario. In future works, the results of the proposed evolutionary approach will be compared with other metaheuristic algorithms such as Particle Swarm Optimization (PSO) and Evolutionary Strategies (ES), among other techniques.

Considering the benefits of the proposed approach, several improvements are proposed as a further work. First, to increase the reliability of the results, a more sophisticated model of the ESS that considers the effects of battery degradation must be considered. In this same line of improvement, the implementation of an electricity price short-term forecasting is also proposed to develop a prize-based UC. Finally, the study of this optimization problem in a multi-objective mode is considered as further work. A multi-objective approach would provide a better understanding of the problem.

Author Contributions: Conceptualization, L.A.-B., A.R.d.N., A.T., J.L.M.-R., D.G.R.; Validation, A.R.d.N., A.T., D.G.R.; Writing original-draft, L.A.-B., A.R.d.N., A.T.; Writing-review and editing, J.L.M.-R., D.G.R.

Funding: Research partially supported by grant TEC2016-80242-P funded by AEI/FEDER through the Laboratorio de Simulación Hardware-in-the-loop de Sistemas Ciberfśicos (LaSSiC), the Spanish Ministry of Economy and Competitiveness under grant PCIN-2015-043 and the University of Seville under the contract "Contratos de acceso al Sistema Español de Ciencia, Tecnología e Innovación para el desarrollo del programa propio de I+D+i de la Universidad de Sevilla

Conflicts of Interest: The authors declare no conflict of interest.

\section{References}

1. Malik, F.H.; Lehtonen, M. A review: Agents in smart grids. Electr. Power Syst. Res. 2016, 131, 71-79. [CrossRef]

2. Kakran, S.; Chanana, S. Smart operations of smart grids integrated with distributed generation: A review. Renew. Sustain. Energy Rev. 2018, 81, 524-535. [CrossRef]

3. Bullich-Massagué, E.; Díaz-González, F.; Aragüés-Peñalba, M.; Girbau-Llistuella, F.; Olivella-Rosell, P.; Sumper, A. Microgrid clustering architectures. Appl. Energy 2018, 212, 340-361. [CrossRef]

4. Soshinskaya, M.; Crijns-Graus, W.H.; Guerrero, J.M.; Vasquez, J.C. Microgrids: Experiences, barriers and success factors. Renew. Sustain. Energy Rev. 2014, 40, 659-672. [CrossRef]

5. Ravichandran, A.; Malysz, P.; Sirouspour, S.; Emadi, A. The critical role of microgrids in transition to a smarter grid: A technical review. In Proceedings of the 2013 IEEE Transportation Electrification Conference and Expo (ITEC), Detroit, MI, USA, 16-19 June 2013; pp. 1-7.

6. Kheradmand-Khanekehdani, H.; Gitizadeh, M. Well-being analysis of distribution network in the presence of electric vehicles. Energy 2018, 155, 610-619. [CrossRef]

7. Parhizi, S.; Lotfi, H.; Khodaei, A.; Bahramirad, S. State of the Art in Research on Microgrids: A Review. IEEE Access 2015, 3, 890-925. [CrossRef]

8. Feng, W.; Jin, M.; Liu, X.; Bao, Y.; Marnay, C.; Yao, C.; Yu, J. A review of microgrid development in the United States-A decade of progress on policies, demonstrations, controls, and software tools. Appl. Energy 2018, 228, 1656-1668. [CrossRef]

9. Hatziargyriou, N.D.; Kleftakis, V.A.; Papadimitriou, C.N.; Messinis, G.M. Microgrids in Distribution. In Smart Grid Handarticle; Wiley Online Library: Hoboken, NJ, USA, 2016; pp. 1-24.

10. Zia, M.F.; Elbouchikhi, E.; Benbouzid, M. Microgrids energy management systems: A critical review on methods, solutions, and prospects. Appl. Energy 2018, 222, 1033-1055. [CrossRef]

11. Elsayed, W.; Hegazy, Y.; Bendary, F.; El-Bages, M. Energy management of residential microgrids using random drift particle swarm optimization. In Proceedings of the 2018 19th IEEE Mediterranean Electrotechnical Conference (MELECON), Marrakech, Morocco, 2-7 May 2018; pp. 166-171.

12. Padhy, N.P. Unit commitment-A bibliographical survey. IEEE Trans. Power Syst. 2004, 19, 1196-1205. [CrossRef] 
13. Nemati, M.; Braun, M.; Tenbohlen, S. Optimization of unit commitment and economic dispatch in microgrids based on genetic algorithm and mixed integer linear programming. Appl. Energy 2018, 210, 944-963. [CrossRef]

14. Lei, X.; Lerch, E.; Xie, C. Frequency security constrained short-term unit commitment. Electr. Power Syst. Res. 2002, 60, 193-200. [CrossRef]

15. Blanco, I.; Morales, J.M. An efficient robust solution to the two-stage stochastic unit commitment problem. IEEE Trans. Power Syst. 2017, 32, 4477-4488. [CrossRef]

16. Wang, Q.; Wang, J.; Guan, Y. Stochastic unit commitment with uncertain demand response. IEEE Trans. Power Syst. 2013, 28, 562-563. [CrossRef]

17. Marneris, I.G.; Biskas, P.N.; Bakirtzis, A.G. Stochastic and deterministic unit commitment considering uncertainty and variability reserves for high renewable integration. Energies 2017, 10, 140. [CrossRef]

18. Khan, A.A.; Naeem, M.; Iqbal, M.; Qaisar, S.; Anpalagan, A. A compendium of optimization objectives, constraints, tools and algorithms for energy management in microgrids. Renew. Sustain. Energy Rev. 2016, 58, 1664-1683. [CrossRef]

19. Reddy, S.; Panwar, L.K.; Panigrahi, B.; Kumar, R.; Alsumaiti, A. Binary grey wolf optimizer models for profit based unit commitment of price-taking GENCO in electricity market. Swarm Evolut. Comput. 2019, 44, 957-971. [CrossRef]

20. Li, P.; Xu, D. Optimal operation of microgrid based on improved binary particle swarm optimization algorithm with double-structure coding. In Proeedings of the 2014 International Conference on Power System Technology, Chengdu, China, 20-22 October 2014; pp. 3141-3146.

21. Bendotti, P.; Fouilhoux, P.; Rottner, C. On the complexity of the unit commitment problem. Ann. Oper. Res. 2019, 274, 119-130. [CrossRef]

22. Che, P.; Shi, G. An MILP approach for a profit-based unit commitment problem with emissions penalty. In Proeedings of the 26th Chinese Control and Decision Conference (2014 CCDC), Changsha, China, 31 May to 2 June 2014; pp. 4474-4477.

23. Zaree, N.; Vahidinasab, V. An MILP formulation for centralized energy management strategy of microgrids. In Proeedings of the 2016 Smart Grids Conference (SGC), Austin, TX, USA, 1-3 July 2016; pp. 1-8.

24. Zhang, D.; Liu, S.; Papageorgiou, L.G. Fair cost distribution among smart homes with microgrid. Energy Convers. Manag. 2014, 80, 498-508. [CrossRef]

25. Wang, M.Q.; Gooi, H. Spinning reserve estimation in microgrids. IEEE Trans. Power Syst. 2011, 26, 1164-1174. [CrossRef]

26. Marquant, J.F.; Evins, R.; Bollinger, L.A.; Carmeliet, J. A holarchic approach for multi-scale distributed energy system optimisation. Appl. Energy 2017, 208, 935-953. [CrossRef]

27. Fu, B.; Ouyang, C.; Li, C.; Wang, J.; Gul, E. An Improved Mixed Integer Linear Programming Approach Based on Symmetry Diminishing for Unit Commitment of Hybrid Power System. Energies 2019, 12, 833. [CrossRef]

28. Cupelli, L.; Schumacher, M.; Monti, A.; Mueller, D.; De Tommasi, L.; Kouramas, K. Simulation Tools and Optimization Algorithms for Efficient Energy Management in Neighborhoods. In Energy Positive Neighborhoods and Smart Energy Districts; Elsevier: Amsterdam, The Netherlands, 2017; pp. 57-100.

29. Guan, X.; Xu, Z.; Jia, Q.S. Energy-efficient buildings facilitated by microgrid. IEEE Trans. Smart Grid 2010, 1, 243-252. [CrossRef]

30. Wang, N.; Zhang, L.; Xie, G. An improved mixed integer quadratic programming algorithm for unit commitment. Dianli Xitong Zidonghua (Autom. Electr. Power Syst.) 2010, 34, 28-32. [CrossRef]

31. Li, H.; Zang, C.; Zeng, P.; Yu, H.; Li, Z. A genetic algorithm-based hybrid optimization approach for microgrid energy management. In Proceedings of the 2015 IEEE International Conference on CYBER Technology in Automation, Control, and Intelligent Systems (CYBER), Shenyang, China, 8-12 June 2015; pp. 1474-1478.

32. Jo, K.H.; Kim, M.K. Stochastic Unit Commitment Based on Multi-Scenario Tree Method Considering Uncertainty. Energies 2018, 11, 740. [CrossRef]

33. Nemati, M.; Bennimar, K.; Tenbohlen, S.; Tao, L.; Mueller, H.; Braun, M. Optimization of microgrids short term operation based on an enhanced genetic algorithm. In Proceedings of the 2015 IEEE Eindhoven PowerTech, Eindhoven, The Netherlands, 29 June-2 July 2015; pp. 1-6. 
34. Askarzadeh, A. A memory-based genetic algorithm for optimization of power generation in a microgrid. IEEE Trans. Sustain. Energy 2018, 9, 1081-1089. [CrossRef]

35. Liang, H.; Gooi, H. Unit commitment in microgrids by improved genetic algorithm. In Proceedings of the 2010 Conference Proceedings IPEC, Singapore, 27-29 October 2010; pp. 842-847.

36. Li, P.; Xu, D.; Zhou, Z.; Lee, W.J.; Zhao, B. Stochastic Optimal Operation of Microgrid Based on Chaotic Binary Particle Swarm Optimization. IEEE Trans. Smart Grid 2016, 7, 66-73. [CrossRef]

37. Quan, H.; Srinivasan, D.; Khosravi, A. Incorporating wind power forecast uncertainties into stochastic unit commitment using neural network-based prediction intervals. IEEE Trans. Neural Netw. Learn. Syst. 2015, 26, 2123-2135. [CrossRef]

38. Ma, H.; Wang, B.; Gao, W.; Liu, D.; Sun, Y.; Liu, Z. Optimal Scheduling of an Regional Integrated Energy System with Energy Storage Systems for Service Regulation. Energies 2018, 11, 195. [CrossRef]

39. Ruth, M.F.; Kroposki, B. Energy systems integration: An evolving energy paradigm. Electr. J. 2014, $27,36-47$. [CrossRef]

40. Lasseter, R.H.; Paigi, P. Microgrid: A conceptual solution. In Proceedings of the 2004 IEEE 35th Annual Power Electronics Specialists Conference (IEEE Cat. No.04CH37551), Aachen, Germany, 20-25 June 2004; Volume 6, pp. 4285-4290. [CrossRef]

41. Hatziargyriou, N.; Asano, H.; Iravani, R.; Marnay, C. Microgrids. IEEE Power Energy Mag. 2007, 5, 78-94. [CrossRef]

42. Shaw, J.J. A direct method for security-constrained unit commitment. IEEE Trans. Power Syst. 1995, 10, 1329-1342. [CrossRef]

43. Fotuhi-Firuzabad, M.; Billinton, R. Unit commitment health analysis in composite generation and transmission systems considering stand-by units. IEE Proc. Gener. Transm. Distrib. 1999, 146, 164-168. [CrossRef]

44. Patel, J.K.; Read, C.B. Handbook of the Normal Distribution; CRC Press: Boca Raton, FL, USA, 1996; Volume 150.

45. Lasnier, F.; Ang, T.G. Photovoltaic Engineering Handarticle; IOP Publishing: Bristol, UK, 1990.

46. Tapia, A.; Tapia, G.; Ostolaza, J.X.; Saenz, J.R. Modeling and control of a wind turbine driven doubly fed induction generator. IEEE Trans. Energy Convers. 2003, 18, 194-204. [CrossRef]

47. Lei, Y.; Mullane, A.; Lightbody, G.; Yacamini, R. Modeling of the wind turbine with a doubly fed induction generator for grid integration studies. IEEE Trans. Energy Convers. 2006, 21, 257-264. [CrossRef]

48. Miller, N.W.; Sanchez-Gasca, J.J.; Price, W.W.; Delmerico, R.W. Dynamic modeling of GE 1.5 and 3.6 MW wind turbine-generators for stability simulations. In Proceedings of the 2003 IEEE Power Engineering Society General Meeting (IEEE Cat. No.03CH37491), Toronto, ON, Canada, 13-17 July 2003; Volume 3, pp. 1977-1983.

49. Deshmukh, M.; Deshmukh, S. Modeling of hybrid renewable energy systems. Renew. Sustain. Energy Rev. 2008, 12, 235-249. [CrossRef]

50. Mohamed, F.A.; Koivo, H.N. System modelling and online optimal management of MicroGrid using Mesh Adaptive Direct Search. Int. J. Electr. Power Energy Syst. 2010, 32, 398-407. [CrossRef]

51. Mohamed, F.A.; Koivo, H.N. Online Management of MicroGrid with Battery Storage Using Multiobjective Optimization. In Proceedings of the 2007 International Conference on Power Engineering, Energy and Electrical Drives, Setubal, Portugal, 12-14 April 2007; pp. 231-236. [CrossRef]

52. Ortega-Vazquez, M.A.; Kirschen, D.S. Estimating the spinning reserve requirements in systems with significant wind power generation penetration. IEEE Trans. Power Syst. 2009, 24, 114-124. [CrossRef]

53. Ortega-Vazquez, M.A.; Kirschen, D.S. Optimizing the spinning reserve requirements using a cost/benefit analysis. IEEE Trans. Power Syst. 2007, 22, 24-33. [CrossRef]

54. Rebours, Y.; Kirschen, D. What is spinning reserve. Univ. Manch. 2005, 174, 175.

55. Azmy, A.; Erlich, I. Online optimal management of PEM fuel cells using neural networks. In Proceedings of the IEEE Power Engineering Society General Meeting, San Francisco, CA, USA, 16 June 2005; Volume 2, p. 1337. [CrossRef]

56. Orero, S.; Irving, M. Large scale unit commitment using a hybrid genetic algorithm. Int. J. Electr. Power Energy Syst. 1997, 19, 45-55. [CrossRef]

57. Wood, A.J.; Wollenberg, B.F. Power Generation, Operation and Control; Wiley Online Library: Hoboken, NJ, USA, 1984. 
58. Arzamendia, M.; Gregor, D.; Reina, D.G.; Toral, S.L. An evolutionary approach to constrained path planning of an autonomous surface vehicle for maximizing the covered area of Ypacarai Lake. Soft Comput. 2019, 23, 1723-1734. [CrossRef]

59. Gutiérrez-Reina, D.; Sharma, V.; You, I.; Toral, S. Dissimilarity Metric Based on Local Neighboring Information and Genetic Programming for Data Dissemination in Vehicular Ad Hoc Networks (VANETs). Sensors 2018, 18, 2320. [CrossRef] [PubMed]

60. Holland, J.H. Genetic Algorithms and Adaptation; Springer: New York, NY, USA, 1984; pp. 317-333. [CrossRef]

61. Luke, S. Essentials of Metaheuristics, 1st ed.; lulu.com: 2009. Available online: http://cs.gmu.edu/\$ sim\$sean/books/metaheuristics/ (accessed on 1 May 2019).

62. Ter-Sarkisov, A.; Marsland, S. Convergence Properties of Two $(\mu+\lambda)$ Evolutionary Algorithms On OneMax and Royal Roads Test Functions. arXiv 2011, arXiv:1108.4080.

63. Deb, K.; Agrawal, R.B. Simulated Binary Crossover for Continuous Search Space. Complex Syst. 1995, 9, 115-148

64. Fortin, F.A.; De Rainville, F.M.; Gardner, M.A.; Parizeau, M.; Gagné, C. DEAP: Evolutionary Algorithms Made Easy. J. Mach. Learn. Res. 2012, 13, 2171-2175.

65. Reina, D. G. Evolutionary Microgrid. 2019. Available online: https://github.com/Dany503/EvolutionaryMicrogrids (accessed on 1 May 2019).

(C) 2019 by the authors. Licensee MDPI, Basel, Switzerland. This article is an open access article distributed under the terms and conditions of the Creative Commons Attribution (CC BY) license (http:/ / creativecommons.org/licenses/by/4.0/). 